\title{
JOURNAL OF ADVANCED MEDICAL AND PHARMACEUTICAL RESEARCH
}

Received $10^{\text {th }}$ June 2020 , Accepted $30^{\text {th }}$ June 2020

jampr.journals.ekb.eg

Online ISSN: 2636-4158

\section{Antibiotic Resistance and Novel Approaches to Fight Against This Global Crisis}

\author{
Tarek Elbanna ${ }^{1}$, Fatma Sonbol', Ahmed Abdelaziz', Engy Elekhnawy ${ }^{1 *}$ \\ ${ }^{I}$ Department of pharmaceutical microbiology, faculty of pharmacy, Tanta university, Tanta, Egypt.
}

\begin{abstract}
Since penicillin was discovered by Sir Alexander Fleming in 1928, antibiotics have been used in medicine to treat different infections and combat pathogenic microorganisms. Nevertheless, with their increasing and massive use, the progress of resistance has become more predominant and many bacteria have developed resistance to multiple classes of antibacterial agents through genetic exchange mechanisms. Consequently, the use of antibiotics is now under threat. Antibiotic resistance poses a worldwide hazard to both human and animal health and it has become an important public health concern in the $21^{\text {st }}$ century. This review aims to survey some mechanisms of antibiotic resistance. Furthermore, some novel approaches like the use of nanoparticle-based antimicrobial drug delivery, fecal microbiota transfer, antimicrobial peptides, antibiotic adjuvants, antisense antimicrobials, phage therapy, antivirulence strategies, host oriented therapy, synthesis of new antimicrobials and extraction of antimicrobials from a natural source like plants are identified and discussed in an attempt to combat and fight against bacterial resistance.
\end{abstract}

Keywords: Antibiotics, Resistance, Bacteria, Enzymatic inactivation, Efflux.

\section{INTRODUCTION}

Antimicrobials are agents that kill microorganisms or inhibit their growth. Antimicrobial agents can be assembled according to the microorganisms they target. For example, antifungals are used against fungi, antibiotics are active against bacteria, antivirals are active against viruses and antiparasitics are active against parasites. ${ }^{1}$ They can also be classified based on their function. Antimicrobials that kill microbes are called cidal and those which inhibit their growth called static. $^{2}$

Antimicrobial drugs are also classified to narrow or broad-spectrum based upon their spectrum of activity. Broad-

*Department of Pharmaceutical Microbiology, Faculty of Pharmacy, Tanta University, Egypt, 31527, Tel: (202) 040-3336007; fax: (202) 040-3335466. Email: Engy.elekhnawy2020@gmail.com spectrum antimicrobials are effective against a wide range of microbes and have higher toxicity to the host. Narrowspectrum antimicrobials are effective against a narrow range of microbes and have lower toxicity to the host. Antimicrobials, especially antibacterials, are classified into chemotherapeutic agents which are obtained from synthetic source to kill or inhibit the growth of the susceptible microorganism and antibiotics which are produced by living organisms, usually fungi. However, antibiotic is frequently used to mean all antibacterials. ${ }^{2}$

\section{MECHANISMS OF ACTION OF ANTIBIOTICS}

An antibiotic is an agent that inhibits bacterial growth or kills bacteria. The term antibiotic was used for the first time in 1942 by Selman Waksman to define any substance produced by a microorganism that is active against the growth of other 
microorganisms. ${ }^{3}$ Many antibacterial agents are classified based on their chemical or biosynthetic origin into natural, semisynthetic, and synthetic. ${ }^{1}$

There are five major modes of action of most antibacterial agents: (a) Interference with cell wall synthesis, (b) Interference with nucleic acid synthesis, (c) Inhibition of protein synthesis, (d) Inhibition of a metabolic pathway, and (e) Disruption of bacterial cell membrane structure. ${ }^{1}$

Antibacterial drugs that inhibit bacterial cell wall synthesis include $\beta$-lactams, like penicillins, cephalosporins, monobactams, and carbapenems and glycopeptides, such as vancomycin and teicoplanin. ${ }^{4} \beta$-Lactams interfere with the enzymes which are required for the synthesis of the peptidoglycan layer so inhibit bacterial cell wall synthesis. ${ }^{5}$ Glycopeptides also interfere with the cell wall synthesis by binding to the terminal $\mathrm{D}$-alanine residues of the nascent peptidoglycan chain, thus preventing the cross-linking required for cell wall synthesis. ${ }^{5}$

\section{BACTERIAL RESISTANCE ANTIBIOTICS}

Some bacterial species are capable of surviving after exposure to one or more antibiotics; and this is called antibiotics resistance. Many bacteria have developed resistance to multiple classes of antibacterial agents through genetic exchange mechanisms and these multidrug-resistant (MDR) bacteria, or superbugs that can be defined as resistance to $\geq$ three antibacterial classes have become an important public health concern in the $21^{\text {st }}$ century, especially in hospitals and other healthcare institutions where they are most commonly detected. ${ }^{9}$

\subsection{Causes of Antibiotic Resistance}

Even though there were low levels of antibiotic-resistant bacteria before the extensive use of antibiotics, misuse and overuse of antibiotics, selective pressure from their usage has played a vital role in the development and the spread of multidrug resistance among bacterial species. ${ }^{10}$

Practices contributing towards resistance include selling antibiotics over the counter without a prescription, addition of antibiotics to the livestock feed, and household use of the antibacterials in soaps and other products. ${ }^{11}$ Unsound practices in the field of pharmaceutical industry can contribute to the likelihood of producing antibiotic-resistant strains as well. ${ }^{12}$

Certain classes of antibiotics are highly associated with colonization with the MDR bacteria compared to other antibiotic classes. The risk for colonization increases if there is a resistance of the MDR bacteria to the antibiotic used and high tissue penetration, in addition to broad-spectrum activity against beneficial bacteria, normal flora of the human body. In the case of Methicillin-resistant Staphylococcus aureus (MRSA), higher rates of MRSA infections are detected with the use of cephalosporins, glycopeptides, and quinolones. ${ }^{13}$ Cephalosporins, quinolones, and clindamycin are the highrisk antibiotics in the case of colonization with Clostridium difficile. ${ }^{14}$

\subsection{Types of Antibiotic Resistance}

There is a variety of mechanisms that have a role in the wide range of sensitivities exhibited by different types of microorganisms when evaluated by sensitivity test assays for determination of minimum inhibitory concentrations (MICs) and minimum bactericidal concentrations (MBCs). Certain microorganisms are intrinsically resistant which is a natural character of all strains of that microorganism. Instead, they may experience changes in the susceptibility due to mutation of the target site, or the acquirement of different genetic elements encoding resistance mechanisms.

\subsubsection{Intrinsic Resistance}

Intrinsic resistance is a natural character of a microorganism and is exhibited by Mycobacteria, bacterial spores, and several Gram-negative bacteria. Mycobateria are even more resistant as they possess a waxy envelope that inhibits the uptake of some antimicrobials. ${ }^{15}$ The coat and the cortex of the bacterial spores are a barrier against the entry of antimicrobials, clarifying their relatively extreme resistance. ${ }^{16}$ The structural and biochemical changes resulting in the germination of the bacterial spores make them more susceptible to the action of some antimicrobials. ${ }^{16}$

Gram-negative bacteria are normally relatively less susceptible to antimicrobials than Gram-positive bacteria due to their outer membrane which acts as a significant barrier to antimicrobial entry. ${ }^{15}, 17$ The outer membrane of Gramnegative bacteria presents a permeable barrier owing to their narrow porin channels that limit the permeation of hydrophobic molecules and the low fluidity of the lipopolysaccharides slows down the inward penetration of different lipophilic compounds. ${ }^{17}$ Consequently, intrinsic microbial resistance is commonly associated with the cellular impermeability that limits the uptake of antimicrobials, even though active efflux pumps seems to be an important process. ${ }^{17}$

Efflux pumps have an important role in the intrinsic resistance of Gram-negative bacteria by pumping out different agents, including detergents and antibiotics. It equips bacteria with a basic and common resistance mechanism by extruding toxic molecules. ${ }^{18}$ The term multidrug resistance (MDR) is used to define a situation where reduced susceptibility to an antibiotic is accompanied by reduced susceptibility to additional chemically unrelated antibiotics by efflux system. ${ }^{18}$ The presence of the efflux mechanisms with the outer membrane narrow porin channels restricts the diffusion of antimicrobials into the cells which are responsible for the high intrinsic resistance of Gram-negative bacteria to many antimicrobials. ${ }^{17}$

In addition to impaired uptake or increased efflux, some microorganisms exhibit intrinsic resistance by inactivation of antimicrobials. Bacterial enzymes can add chemical groups to exposed sites of the antibiotic molecule leading to prevention of the antibiotic from binding to its target site. ${ }^{19}$ Hydroxyl and amides groups of antibiotics can easily be hydrolyzed leading to an alteration in the antibiotic 
structures. Furthermore, phosphate, acetyl, and nucleotide groups can be added to the antibiotics rendering them inactive. $^{20}$

Phenotypic (physiological) adaptation of microorganisms that reduces susceptibility to antimicrobials in response to changes in the environmental conditions is also considered as intrinsic resistance. ${ }^{15,21}$ The cellular phenotype expressed differ significantly with the changes in the environmental conditions under which it is grown. ${ }^{22,23}$ The resistance of these microorganisms to antimicrobials may result partially from outer cell layers changes that increase the barrier properties and inhibit access to their target site; however other changes can also be involved. ${ }^{6}$

The binding of microorganisms to solid surfaces results in the formation of a biofilm. ${ }^{15}, 22,24$ Reduced susceptibilities of bacteria in embedded in biofilms to different antimicrobials can sometimes be very high and can be triggered by variable factors including depletion of nutrient within the biofilm, reduced entry of the antimicrobial agent to the cells present in the biofilm, possible chemical interaction between the antimicrobial agent and the biofilm, and the production of enzymes that degrade the antimicrobial agent. ${ }^{24}$ Biofilms have been found that they possess susceptibilities towards antimicrobial agents that are 100-1000 times lower than equivalent populations of planktonic bacteria; bacteria not forming biofilm. ${ }^{25}$

\subsubsection{Acquired Resistance}

The ability of bacteria to survive in the existence of antibiotics and to gain resistance under selective pressures exerted by antibiotics assumes that the antibiotics threshold concentration is necessary to induce and sustain resistance genotypes. ${ }^{26}$ Acquired resistance usually arises owing to the pressures from the surrounding microbiome; however, acquired resistance is a reflection of the microbe ecological niche. It is progressively evident that there are many reservoirs of antibiotics in the environment able to enrich the antibiotic-resistant microorganisms. ${ }^{27}$ Therefore, acquired resistance includes genetic changes environmentally induced like biofilm and persister development, changes in the target of the antibiotic, enzymatic inactivation of antibiotics, efflux pump regulation, and changes in the cell permeability. ${ }^{28,29}$

\subsection{Genetic Basis of Antibiotic Resistance}

Regardless of whether the antimicrobial resistance is intrinsic or acquired, several different mechanisms of it exist.

\subsubsection{Horizontal Gene Transfer (HGT)}

The majority of the acquired antibiotic resistance spreads through horizontal or lateral gene transfer between bacteria of the same generation often because of the polymicrobial nature of different infections and closeness of the pathogenic microorganisms. ${ }^{29}$ Horizontal gene transfer can occur in one of three ways: (1) Infection by bacteriophages (transduction), (2) uptake of environmental DNA (transformation) or
(3) exchange of plasmids (conjugation). ${ }^{30}$ Nevertheless, this transfer of genetic material does not occur without an evolutionary cost. ${ }^{31}$ To reduce this evolutionary cost, bacteria have established numerous genetic strategies like plasmids, transposons, operons, and gene clusters. ${ }^{29,} 32$ The ability to move the genetic material between bacterial species may explain the transfer of antibiotic resistance and the extension of resistance beyond a single drug. ${ }^{29}$

\subsubsection{Elevated Mutation Rates}

Despite the fitness costs, antibiotic pressure selects for bacteria which have a high mutation rate. ${ }^{33}$ Mycobacteria can acquire resistance via mutation under inadequate treatment regimens as are Salmonella enterica, Escherichia coli, and Pseudomonas aeruginosa. ${ }^{27}$ This increased mutation rate is conferred by changes in the genes which constitute the mismatch repair (MMR) system (mutS, mutL, mutH, mutT, mutY, mutM, and uvrD). ${ }^{29}$ Mutations occurring in the MMR system increase the prevalence of genetic recombination, so it provides diversity to the mechanisms of antibiotic resistance. ${ }^{33}$ Away from the heritable genetic alterations to mismatch repair systems, ${ }^{34}$ antibiotics can increase the mutation rates by the stress responses. ${ }^{26}$

\subsection{Mechanisms of Antibiotic Resistance}

Antibiotic resistance has an important role in the failure of the treatment of different infectious diseases. ${ }^{35}$ There are several mechanisms of resistance that spread to various bacterial genera. First, the microorganism can acquire genes that encode enzymes, like $\beta$-lactamases, which destroy the antibacterial drug before it can produce an effect. Second, bacteria can have efflux pumps that extrude the antibacterial drug from the bacterial cell before it reaches its target site and produces its effect. Third, bacteria can acquire genes that ultimately produce changed bacterial cell walls with no binding site for the antimicrobial drug, or bacteria can acquire mutations which limit the access of antibacterial agent to its intracellular target site by downregulation of the porin genes. Therefore, populations of bacteria that are normally susceptible may become resistant to antibiotics through selection and mutation, or by acquiring various genes that encode resistance from other bacteria. ${ }^{36}$

Bacteria may exhibit any of these four general mechanisms of antibiotic resistance: (a) Lack of entry and decreased cell permeability, (b) Greater exit and active efflux, (c) Enzymatic inactivation/ modification of the antibiotic, (d) Altered target and modification of antibiotic target site.

\subsubsection{Prevention of antibiotic entry}

In addition to the cell membrane which acts as a barrier to antibiotic entry, antibiotics have to pass barriers of cell envelope such as the outer membrane in Gram-negative bacteria to be able to reach their targets. Changes that affect the permeability of these barriers, like the number of outer membrane porins and/or lipopolysaccharides, can affect the resistance against antibiotics. ${ }^{37,38}$ Many antibiotics (like $\beta$ - 
lactams) are transported across the outer membrane of the Gram-negative by a group of membrane proteins, called porins. Several factors like the charge and the hydrophobicity of the antibiotic have a significant role in its transport into the cell. Molecules having negative charge pass more slowly across the cell membrane than do the positively charged molecules or zwitterions. ${ }^{39}$

On the other hand, polycations, such as polymyxin $\mathrm{B}$, which are larger than the exclusion limit of the outer membrane can enter the cell membrane by a pathway called the self-promoted uptake. In this pathway, the polycations competitively displace the divalent cations that cross-bridge the adjacent lipopolysaccharides (LPS), so disrupt the outer membrane. $^{40}$

\subsubsection{Active extrusion of antibiotics}

Several integral membrane proteins mediate active antibiotic extrusion leading to lower cytoplasmic antibiotic concentration and an increase in antibiotic resistance. ${ }^{41}$ Efflux systems in bacteria can be divided into four families based on supramolecular assembly, mechanism and sequence homology: (i) resistance nodulation division (RND) family, (ii) major facilitator (MF) family, (iii) staphylococcal multidrug resistance (SMR) family, and (iv) adenosine triphosphate (ATP)-binding cassette (ABC) transporters. ${ }^{9}$

\subsubsection{Enzymatic inactivation or degradation of antibiotics}

Drug inactivation is the major mechanism of resistance towards $\beta$-lactam antibiotics. Inactivation of $\beta$-lactam antibiotics like penicillin is mediated by penicillinase enzyme that catalyzes the hydrolysis of the $\beta$-lactam ring. Other wellknown enzymes that cause drug inactivation are chloramphenicol transferases and aminoglycoside modifying enzymes. Accumulation of antibiotics at their site of action in the bacterial cell is the sum of transport into the cell, inactivation during the transport process, and the extrusion of antibiotics from the cell. ${ }^{42}$

\subsubsection{Alteration of antibiotic target}

Protection by alteration of the antibiotic targets may prevent the interaction and hence the toxicity of antibiotics. Alteration of the antibiotic targets (such as amino acid substitutions) decreases the affinity for the antibiotic. Penicillin resistance results from alterations in the penicillin-binding proteins (PBPs) which form bind irreversibly with penicillin, resulting in loss of the penicillin activity. ${ }^{43}$

\subsection{Strategies of Control Antibiotic Resistance}

First, the rational use of antibiotics is very important to reduce the chances of occurrence of opportunistic infections caused by antibiotic-resistant bacteria. The immune system can cure minor bacterial infections on its own. If it is given the chance without depending on antibiotics to cure the small infections, resistance to antibiotics will be less likely to occur.
Additionally, it is important to be noted that antibiotics are unable to treat viral infections. Taking antibiotics unnecessarily to cure viral infections can lead to the spread of resistance to antibiotics. ${ }^{44}$

Second, the simultaneous use of two or more antibiotics separately or in a single pharmaceutical formulation is called antibiotic combination. The advantages of combination therapy include avoiding the development of resistance and decreasing the toxicity of the individual antibiotic as they are used in lower doses, providing broad coverage in poly-microbial infections and treatment of severe infections. $^{45}$

The third strategy which relies on the infection control practices that must be applied in hospitals and different health care institutions to limit nosocomial infections in patients and staff members. These practices like correct handling procedures for contaminated materials to decrease the transmission of microorganisms. Also, good hand decontamination for health-care workers can be done with soap and water or antiseptic solution. Similarly, sterilization and disinfection of the patient care equipment are essential. ${ }^{46}$, 47

Finally, the restriction of certain antibiotic classes can be effective in changing the patterns of resistance in individual institutions (a process called antibiotic restriction policy). ${ }^{48}$

\section{NEW APPROACHES TO FACE THE SPREAD OF ANTIBIOTIC RESISTANCE}

\subsection{Nanoparticle-Based Antimicrobial Drug Delivery}

Recently, the field of antimicrobial drug delivery has accomplished important progress, particularly with the rapid development of nanomedicine along with the increased understanding of different infectious diseases. Great efforts have been done to increase the therapeutic efficacy of antibiotics with decreasing resistance using nanoparticlebased strategies. ${ }^{49}$ These strategies have shown hopeful outcomes by localization of the antibiotics to the pathogen and modulation of the drug-pathogen interaction against bacterial drug resistance. ${ }^{50}$ Although clinical uses of antimicrobial nanoparticles are still scarce, their development is very fast. For instance, inorganic nanoparticles such as $\mathrm{Ag}$ and $\mathrm{ZnO}$ are producing a remarkable comeback as effective antimicrobial agents. ${ }^{51}$ The success of the antimicrobial nanoparticles suggests that they will have a promising future for curing different infections. It is expected that the field of nanomedicine will continue to produce innovations tailored for the local antimicrobial treatment having advantages of efficiency, cost-effectiveness, and patient compliance. ${ }^{49}$

\subsection{Antimicrobial Agents from Natural Source}

Since ancient times, products of both plants and bees have been used empirically to cure different infections. Nevertheless, intensive research is required to study the 
molecular mechanisms of action of such compounds. The suggested mechanisms of antimicrobial action of the essential oils extracted from plants include the degradation of cell walls; increased permeability due to cytoplasmic membrane damage; membrane protein damage, leading to decreasing the proton-motive force and cytoplasm coagulation..$^{52}$

It is urgent to search for novel and/or renewable natural sources for the fight against antibiotic-resistant bacterial infections. Soon, marine-derived antimicrobials, such as secondary metabolites of actinomycetes, marine fungi polyketides, and cyanobacterial alkaloids could become a powerful tool for the control and prevention of microbial resistance as they can be used as alternatives for the currently used antibiotics. ${ }^{52,53}$

\subsection{Antivirulence Strategies}

Inhibition of quorum sensing is an important example of antivirulence strategies. Quorum sensing (QS) is communication between bacterial cells that has been found to occur between bacterial cells of the same and different species and between bacteria and higher organisms. ${ }^{54}$ Bacteria must possess three characteristics for QS to occur: 1) secrete a signaling molecule (autoinducer or pheromones) that are produced in response to the changes in the density of cellpopulation, 2) detect the change in the concentration of pheromones, and 3 ) regulate the gene of transcription. ${ }^{55}$

Quorum quenching or anti-quorum sensing is the process of prevention of quorum sensing by disruption of the signalling. This is accomplished by inactivating the signalling enzymes involved in the synthesis of autoinducers (like triclosan), also by introducing molecules that mimic signalling molecules and block their receptors (like furanones), or by degrading the signalling molecules. ${ }^{56}$

\subsection{Phage Therapy}

It is the therapeutic use of bacteriophages to cure different bacterial infections. Bacteriophages are more specific than antibiotics so, they are harmless to the host organism and beneficial bacteria like gut flora. However, this specificity is also a disadvantage because a phage will only kill one type of bacterial species. Therefore, phage mixtures should be used to increase the chances of success of the treatment. ${ }^{57}$

As phages replicate only in vivo, a smaller effective dose could be used. ${ }^{57}$ Phages are more successful than antibiotics when the bacteria are embedded in a biofilm, as antibiotics typically cannot penetrate the polysaccharide matrix of the biofilm. Phage therapy offers a probable alternative to the traditional antibiotics used for the treatment of bacterial infection. Although bacteria can evolve resistance to phages, the resistance is easier to overcome than the resistance to antibiotics. Just as bacteria can develop resistance, viruses can be manipulated to overcome this resistance. $^{58}$

Phage therapy is also safe, as featured by its widespread clinical use in the former Soviet Union where it was applied before the antibiotic era. ${ }^{59,60}$

\subsection{Antisense Antimicrobials}

Antisense antimicrobials are synthetic oligomers that silence the expression of certain genes. This specificity provides an advantage over the broad-spectrum antibiotics by avoiding unwanted effects on commensal bacteria. The specificity of the sequence of the antisense antimicrobials used and its short length pose little risk to the human gene expression. ${ }^{61}$ Antisense antimicrobials can be rapidly designed and synthesized to target virtually any microbe as they are a platform technology. This decreases the drug discovery time and offers flexibility in drug development. Recently, it has been shown that antisense technology has the potential to overcome the antibiotic resistance crisis, as the resistance mechanisms for the standard antibiotics have no impact on antisense antimicrobials. ${ }^{62}$

\subsection{Host-Oriented Therapy}

Host-oriented therapy including vaccination, immunotherapy, and immunomodulation has a great advantage of possessing a very low probability to develop resistance. Artificial immunity can be actively acquired, either by vaccination (active immunity) or by the transfer of exogenous antibodies (passive immunity). ${ }^{63}$ The purpose of vaccination is to eradicate, eliminate, or limit an infectious process. ${ }^{64}$ Immunotherapy involves the administration of certain antibodies that can neutralize the action of its corresponding antigens. The increasing rate of antimicrobial resistance has refocused the research attention to the use of immunomodulators which can interact with the nonspecific and specific immune cells and change their reactivity towards antigens, via the cytokine signaling pathways. ${ }^{65}$

\subsection{Antimicrobial Peptides}

Antimicrobial peptides are antimicrobial proteins synthesized in the ribosome and then released extracellularly. These compounds are formed by many bacterial species and can kill or inhibit the growth of other bacteria. It was found that these compounds have great potential against antibiotic-resistant bacterial isolates. Bacteriocins are an example of antimicrobial peptides and they are produced by both Grampositive and Gram-negative bacteria. ${ }^{66}$ Bacteriocins could be an efficient new class of antibiotics having the advantages of specific targets, safety (food grade), diversity, reduced cell toxicity, and ability to act synergistically with the traditional antibiotics. Nevertheless, they may be susceptible to proteolytic enzymes and some of them may become toxic to mammalian cells. Currently, bacteriocins have been broadly used, especially in the food industry. ${ }^{67}$

\subsection{Antibiotic Adjuvants (Resistance Breakers and Antibiotic Potentiators)}

The use of antibiotic adjuvants is an approach that should receive more attention. These are non-antibiotic agents when co-administered with antibiotics, block the resistance 
(resistance breakers) or enhance the antimicrobial activity (potentiators). $\beta$-lactamase inhibitors are an example of antibiotic adjuvants which are being used successfully in the medical field for almost 30 years. They block the activity of $\beta$-lactamase enzymes which break down $\beta$-lactam antibiotics causing resistance to $\beta$-lactams. ${ }^{68,69}$

\subsection{Synthesis of New Antibiotics}

The introduction of new functional groups in the existing antibiotic molecule to produce new antibiotics with a dual or triple mechanism of action can be done. Also, the synthesis of novel dual-action antibiotics by binding two different antibiotic molecules (called hybrid antibiotic) can be studied. $^{70}$ Moreover, extensive research is needed for the synthesis of new antibiotics with unique characters such as new or improved mechanism of action, new physicochemical property to avoid its recognition by the bacterial efflux pumps, ability to survive the action of the inactivating enzymes produced by the resistant bacteria and ability to bind to the altered target in the resistant bacteria. ${ }^{70}$

\subsection{Fecal Microbiota Transplant (FMT)}

Fecal microbiota transplant (FMT), or stool transplant, is the process of transplantation of the fecal bacteria from a healthy individual into a recipient. ${ }^{71}$ FMT implicates the restoration of the microflora of the colon by infusion of stool containing healthy bacterial flora by colonoscopy or orogastric tube or enema or through a capsule containing freeze-dried material administered orally. The effectiveness of FMT has been recognized in clinical trials for the treatment of infection caused by Clostridium difficile that causes pseudomembranous colitis. $^{72,73}$

\section{CONCLUSION}

The discovery of antibiotics can be considered as one of the most important achievements in medicine. The use of antibiotics has improved clinical consequences from infections, resulting in the reduction of the morbidity and mortality in surgical, transplant, and critical care patients. Unfortunately, with the extensive use of broad-spectrum antibiotics, selective pressures have made the antibiotic resistance phenomenon an urgent global concern. The percentages of hospital-acquired infections caused by multidrug-resistant bacteria are continuously increasing, making treatment more difficult and antibiotic choice progressively limited. It is time to find alternatives to traditional antibiotic treatments. In this review we tried to present some of the options available and more research is highly needed to win in our battle against antibiotic-resistant bacteria.

\section{CONFLICTS OF INTEREST}

The authors declare no conflict of interest.

\section{REFERENCES}

1. M. A. Kohanski, D. J. Dwyer and J. J. Collins, Nat. rev. Microbiol., 2010, 8, 423-435.

2. J. H. Powers, CBI, 2004, 10, 23-31.

3. D. Julian and D. Davies, $M M B R, 2010,74,417-33$.

4. G. Kapoor, S. Saigal and A. Elongavan, JOACP, 2017, 33, 300-305.

5. H. Cho, T. Uehara and T. G. Bernhardt, Cell, 2014, 15, 1300-1311.

6. R. J. Fair and Y. Tor, Perspect. Medicin. Chem., 2014, 6, 25-64.

7. L. Poirel, A. Jayol and P. Nordmann, Clin. Microbiol. Rev., 2017, 30, 557-596.

8. A. Müller, M. Wenzel, H. Strahl, F. Grein, T. N. V. Saaki, B. Kohl, T. Siersma, J. E. Bandow, H.-G. Sahl, T. Schneider and L. W. Hamoen, Proceedings of the National Academy of Sciences, 2016, 11, 77-86.

9. H. Nikaido, Ann. Rev. Biochem., 2009, 78, 119-146.

10. C.L. Ventola, J. Formul. Manag., 2015, 40, 277-83.

11. K. Landfried, L. Barnidge, E. Pithua, P. Lewis, R. Jacoby, C. King and C. Baskin, Animals, 2018, 8, 198209.

12. D.G. Larsson and J. Fick, Regul. Toxicol. Pharmacol., 2009, 53, 161-169.

13. E. Tacconelli, G. De Angelis, M.A. Cataldo, E. Pozzi and R. Cauda, J. Antimicrob. Chemother., 2008, 61, 26-38.

14. E. J. Kuijper, J. Van Dissel and M.H. Wilcox, Curr. Opin. Infect. Dis., 2007, 20, 376-83.

15. G. McDonnell and A.D. Russell, Clin. Microbiol. Rev., 1999, 12, 147-179.

16. I.M. Gut, S.R. Blanke and W.A. van der Donk, Chem. Biol., 2011, 6, 744-752.

17. H.I. Zgurskaya, C. A. Löpez and S. Gnanakaran, Infect. Dis., 2015, 1, 512-522.

18. X.Z. Li, P. Plésiat and H. Nikaido, Clin. Microbiol. Rev., 2015, 28, 337-418

19. J.M. Blair, M.A. Webber and A.J. Baylay, Nat. Rev. Microbiol., 2014, 13, 42-51.

20. M. Mingeot-Leclercq and J. Décout, Med. Chem. Comm., 2016, 7, 586-611.

21. F. Corona and J.L. Martinez, Antibiot., 2013, 2, 237255.

22. A.D. Russell, Lancet Infect. Dis., 2003, 3, 794-803.

23. T. Elbanna, A. Abdelaziz, F. Sonbol and E. Elekhnawy, Mol. Biol. Rep., 2019, 46, 3437-3443.

24. R. Huang, M. Li and R.L. Gregory, Virulence, 2011, 2, 435-444.

25. P. She, Y. Wang and Z. Luo, Microbiol. Open., 2018, 7, 545-560

26. J.C. Kester and S.M. Fortune, Crit. Rev. Biochem. Mol. Biol., 2013, 49, 91-101.

27. D.I. Andersson and D. Hughes, Drug Resist. Updat., 2012, 15, 162-172.

28. A. Abdelaziz, F. Sonbol, T. Elbanna and E. Elekhnawy, Microb. Drug Resist., 2018, 25, 631-638.

29. M. Schroeder, B.D. Brooks and A.E. Brooks, Genes, 2017, 8, 1-23. 
30. C.J. von Wintersdorff, J. Penders and J.M. van Niekerk, Front. Microbiol., 2016, 7, 173-180.

31. S. Gandon and P.F. Vale, J. Evol. Biol., 2013, 27, 303312

32. X.Z. Li and H. Nikaido, Drugs, 2009, 69, 1555-1567.

33. A. Rodríguez-Rojas, J. Rodríguez-Beltrán, A. Couce and J. Blázquez, Int. J. Med. Microbiol., 2013, 303, 293-297.

34. A.A. Rushdy, M.I. Mabrouk, F.A. Abu-sef, Z.H. Khiralla, S.M. Abdel-All and N.M. Saleh, Braz. J. Infect. Dis. 2013, 17, 431-437.

35. V. Francoise, L. Elisabettea and M. Poul, Biochem. Pharmacol. 2000, 4, 457-4769.

36. F.C. Tenover, Americ. J. Med., 2006, 119, S3-S10.

37. S. Stafani and A. Agodi, Antimicrob. Agents Chemother., 2000, 13, 143-159.

38. F. Sonbol, T. Elbanna, A. Abdelaziz and E. Elekhnawy, J. Appl. Microbiol., 2018, 126, 730-739.

39. U. Choi and C.R. Lee, Front. Microbiol., 2019, 10,953965.

40. L. Zhang, P. Dhillon, H. Yan, S. Farmer and R.E. Hancock, Antimicrob. Agents Chemother., 2000, 44, 3317-3321.

41. S.P. Bernier and M.G. Surette, Front. Microbiol., 2013 , 4, 20-37.

42. K.F. Kong, L. Schneper and K. Mathee, APMIS, 2010, 118, 1-36.

43. S. Sun, M. Selmer and D.I. Andersson, PLoS One, 2014, 9, e97202.

44. M.E. McCusker, A.D. Harris, E. Perencevich and M.C. Roghmann, Emerging Infect. Dis., 2003, 9, 730-733.

45. S. Leekha, C.L. Terrell and R.S. Edson, Mayo Clin Proc., 2011, 86, 156-167.

46. Y. Mehta, A. Gupta, S. Todi and S. Myatra, Ind. J. Crit. Care Med., 2014, 2014, 149-163.

47. A. Nazir and S.M. Kadri, Int.J.Res. Med.Sci., 2014, 2, 21-27.

48. K. de With, F. Allerberger and S. Amann, Infect., 2016, 44, 395-439.

49. N. Beyth, Y. Houri-Haddad, A. Domb, W. Khan and R. Hazan, J. Evidence-Based Integr. Med., 2015, 2015, 24-33.

50. L. Zhang, D. Pornpattananangku, C.M. Hu and C.M. Huang, Curr. Med. Chem., 2010, 17, 585-94.

51. Y.M. Baek and Y.J. An, Sci. Total Environ., 2011, 409,1603-8.

52. H. Chandra, P. Bishnoi, A. Yadav, B. Patni, A.P. Mishra and A.R. Nautiyal, Plants, 2017, 6, 16-30.

53. S. Agrawal, D. Acharya, A. Adholeya, C.J. Barrow and S.K. Deshmukh, Front. Pharmacol., 2017, 8, 828-840.

54. S.T. Rutherford and B.L. Bassler, Perspect. Med., 2012, 2, 12-27.

55. S.A. Paddera, R. Prasadb and A.H. Shah, Microbiol. Res., 2018, 210, 51-58.

56. F. Nazzaro, F. Fratianni and R. Coppola, Inter. J. Mol. Sci., 2013, 14, 12607-12619.

57. D.M. Lin, B. Koskella and H.C. Lin, J. Gastrointest. Pharmacol. Therap., 2017, 8, 162-173.
58. C. Hill, S. Mill and R. Ross, Fut. Microbiol., 2018, 13, $1-12$.

59. P. Hyman, Pharmaceut., 2019, 12, 35-55.

60. V.V. Morozova, Y.N. Kozlova, D.A. Ganichev and N.V. Tikunova, Meth. Mol. Biol., 2018, 1693, 151-158.

61. E.K. Sully and B.L. Geller, Curr. Opin. Microbiol., 2016, 33, 47-55.

62. J. Singh, H. Kaur, A. Kaushik and S. Peer, Inter. J. Pharmacol., 2011, 7, 294-315.

63. T.M. Clay, T. Osada, Z.C. Hartman, A. Hobeika, G. Devi, M.A. Morse and H.K. Lyerly, Immun. Res., 2011, 49, 235-247.

64. P. Klepac, C.J. Metcalf, A.R. McLean and K. Hampson, Biol. Sci., 2013, 368, 201-237

65. J. Munguia and V. Nizet, Trends Pharmacol. Sci., 2017, 38, 473-488.

66. A.A. Bahar and D. Ren, Pharmaceut., 2013, 6, 1543 1575.

67. S.C. Yang, C.H. Lin, C.T. Sung and J.Y. Fang, Front. Microbiol., 2014, 5, 241-268.

68. P. Bernal, C. Molina-Santiago, A. Daddaoua and M.A. Llamas, Microbial. Biotech., 2013, 6, 445-449.

69. C. González-Bello, Bioorganic Medicin. Chem. Lett., 2017, 27, 4221-4228

70. A. Zhivich, Microbiology Independent Research, 2017, 4, 31-51.

71. S. Gupta, E. Allen-Vercoe and E.O. Petrof, Therap. Advan. Gastroenter., 2016, 9, 229-239.

72. L.P. Smits, K.E. Bouter, W.M. de Vos, T.J. Borody and M. Nieuwdorp, Gastroenter., 2013, 145, 946-53.

73. N. G. Rossen, S. Fuentes, M.J. van der Spek and J.G. Tijssen, Gastroenter., 2015, 149, 110-118. 